\title{
Investigations of the few-nucleon systems within the LENPIC project
}

\author{
Jacek Golak $^{1 \star}$, Evgeny Epelbaum ${ }^{2}$, Alessandro Grassi ${ }^{1}$, Hiroyuki Kamada ${ }^{3}$, \\ Hermann Krebs $^{2}$, Patrick Reinert ${ }^{2}$, Roman Skibiński ${ }^{1}$, Volodymyr Soloviov ${ }^{1}$, \\ Kacper Topolnicki ${ }^{1}$, Vitalii Urbanevych ${ }^{1}$, Yuriy Volkotrub ${ }^{1}$ and Henryk Witała ${ }^{1}$
}

1 M. Smoluchowski Institute of Physics, Jagiellonian University, PL-30348 Kraków, Poland

2 Ruhr-Universität Bochum, Fakultät für Physik und Astronomie, Institut für Theoretische Physik II, D-44780 Bochum, Germany

3 Department of Physics, Faculty of Engineering, Kyushu Institute of Technology, Kitakyushu 804-8550, Japan

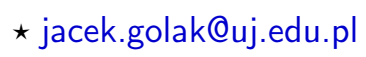

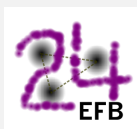

Proceedings for the 24th edition of European Few Body Conference,

Surrey, UK, 2-6 September 2019

doi:10.21468/SciPostPhysProc.3

\begin{abstract}
Results presented in this contribution are obtained within the Low Energy Nuclear Physics International Collaboration (LENPIC). LENPIC aims to develop chiral nucleon-nucleon and many-nucleon interactions complete through at least the fourth order in the chiral expansion. These interactions will be used together with consistently derived current operators to solve the structure and reactions of light and medium-mass nuclei including electroweak processes. In this contribution the current status of the chiral nuclear forces and current operators will be briefly discussed. A special role played by the calculations of nucleon-deuteron scattering will be explained.
\end{abstract}

(c) (i) Copyright J. Golak et al.

This work is licensed under the Creative Commons

Attribution 4.0 International License.

Published by the SciPost Foundation.
Received 07-10-2019

Accepted 05-12-2019

Published 24-02-2020

doi:10.21468/SciPostPhysProc.3.002

\section{Introduction}

The Low Energy Nuclear Physics International Collaboration (LENPIC) is a project established in 2013 that "aims to develop chiral effective field theory nucleon-nucleon (NN) and threenucleon $(3 \mathrm{~N})$ interactions complete through at least fourth order in the chiral expansion (N3LO). Using these new interactions, LENPIC aims to solve the structure and reactions of light nuclei including electroweak observables with consistent treatment of the corresponding exchange currents" [1]. This initiative has brought together physicists from several institutions: Ruhr-University Bochum, Germany, University of Bonn, Germany, Technical University of Darmstadt, Germany, Jagiellonian University, Cracow, Poland, Iowa-State University, USA, Jülich Research Centre, Germany, Kyushu Institute of Technology, Japan, Ohio State University, 
USA, Orsay Institute of Nuclear Physics, France, and TRIUMF, Canada. The Co-Spokespersons of this collaboration are Evgeny Epelbaum from Ruhr-University Bochum and James Vary from Iowa State University.

\section{Nucleon-deuteron scattering with semi-phenomenological nu- clear potentials}

Each group involved in the LENPIC project contributes its own expertise. The Cracow-Bochum group had a lot of experience related to investigations of elastic nucleon-deuteron scattering and nucleon-induced deuteron breakup processes. These investigations, based on rigorous solutions of the 3N Faddeev equations in momentum space started in the 1980s and in the 1990s were carried out with the semi-phenomenological (the so-called "realistic") NN forces, which very accurately described the properties of the two-nucleon (2N) system. These were the AV18 [2], CD Bonn [3], NijmI, NijmII, Nijm93 and Reid93 [4] potentials. The results of these studies (see for example Refs. [5,6]) proved that in general predictions for $3 \mathrm{~N}$ scattering observables agree well with data at the incoming nucleon energies below approximately 30 $\mathrm{MeV}$.

The situation was different at higher energies, where clear discrepancies between the theoretical predictions employing $\mathrm{NN}$ forces only and data emerged. For the minimum of the elastic scattering cross section agreement with the data was restored, at least for energies below approximately $140 \mathrm{MeV}$, when the Tucson-Melbourne [7] or Urbana IX [8] 3N force (3NF) models were added to the $3 \mathrm{~N}$ Hamiltonian. The parameters of the $3 \mathrm{~N}$ potentials used in the calculations were chosen to reproduce the experimental triton binding energy $[6,9,10]$. On the other hand, for many spin observables in elastic nucleon-deuteron scattering (for example the nucleon analyzing power and the deuteron tensor analyzing powers $[6,11]$ ) large $3 \mathrm{NF}$ effects were predicted, but the available combinations of $2 \mathrm{~N}$ and $3 \mathrm{~N}$ forces could not reproduce the data. One of possible reasons for this disagreement between the theoretical predictions and the data could be the nonrelativistic character of the formalism. However, the results obtained within the framework of relativistic Faddeev equation $[12,13]$ revealed only small effects in the cross section. Also elastic scattering polarization observables are only slightly affected by relativity at the considered energies $[12,13]$.

The conclusion from all these studies was that the discrepancies observed at higher energies, which persisted even when the Tucson-Melbourne or Urbana IX 3NF models were included in the calculations, called for better models of the 3NF (with a richer spin structure) and consistence between the $2 \mathrm{~N}$ and $3 \mathrm{~N}$ potentials. This could be achieved only within the chiral effective field theory.

\section{Nucleon-deuteron scattering with chiral nuclear potentials}

In [14] for the first time that consistency was realized when low energy $3 \mathrm{~N}$ scattering was investigated with chiral next-to-next-to-leading order (N2LO) $2 \mathrm{~N}$ and $3 \mathrm{~N}$ forces. Later in Refs. $[15,16]$ precise $2 \mathrm{~N}$ potentials were derived at next-to-next-to-next-to-leading order (N3LO) of the chiral expansion. They reproduced experimental phase-shifts $[17,18]$ in a wide energy range and almost equally well as the semi-phenomenological $2 \mathrm{~N}$ potentials. The corresponding N3LO 3N force contributions were derived in Refs. $[19,20]$. They do not involve any unknown parameters. The large number of terms in the 3NF at N3LO required a new automatized method of their partial-wave decomposition [21-23]. The two free parameters of the $3 \mathrm{~N}$ potential at N2LO were fixed to reproduce the triton binding energy and the nucleon- 

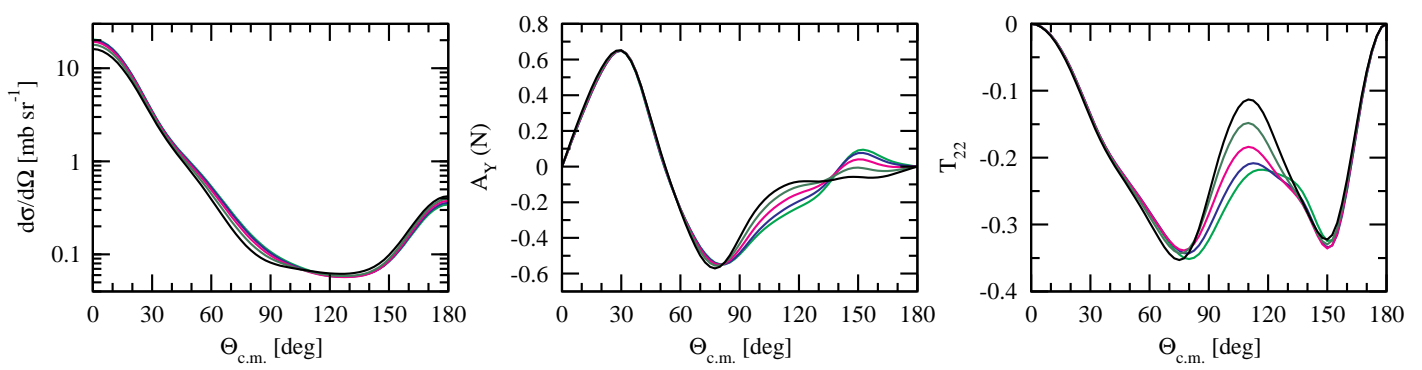

Figure 1: The differential cross section $d \sigma / d \Omega$ (left panel), the nucleon analyzing power $A_{y}(N)$ (middle panel) and the deuteron tensor analyzing power $T_{22}$ (right panel) for elastic nucleon-deuteron scattering at the laboratory nucleon energy $E=$ $200 \mathrm{MeV}$ as a function of the center of mass nucleon scattering angle $\theta_{\text {c.m. }}$ calculated with the chiral N4LO $2 \mathrm{~N}$ potential $[25,26]$ employing the local coordinate-space regularization. The five lines show predictions obtained with five different regulator values $R=0.8,0.9,1.0,1.1$ and $1.2 \mathrm{fm}$.

deuteron doublet scattering length. Both the $2 \mathrm{~N}$ and $3 \mathrm{~N}$ forces required regularization, which in this first generation of the chiral forces was realized by simple non-local partial wave independent regulators, applied in momentum space. Unfortunately, the use of such non-local regularization was the source of finite-cutoff artefacts in the results for higher-energies elastic nucleon-deuteron scattering [24]. These artefacts appear for all non-locally momentum-space regularized $2 \mathrm{~N}$ potentials and are a major obstacle to employing such forces in $3 \mathrm{~N}$ continuum calculations.

A new improved generation of $2 \mathrm{~N}$ chiral potentials prepared up to next-to-next-to-nextto-next-to-leading order (N4LO) [25, 26] employed a local coordinate-space regularization of the one- and two-pion exchange contributions to the $2 \mathrm{~N}$ force. The change leads to a significant reduction of the finite-cutoff artefacts for high energy observables, especially for the differential cross section. This effect is shown in Fig. 1 and should be compared with Fig. 12 from Ref. [24]. (Note that further significant reducion of the finite cutoff artefacts, also for the polarization observables, is achieved in the calculations employing the newest chiral NN potentials from Ref. [30] described below.) These findings are consistent with the ones obtained in the two-nucleon sector $[25,26,30]$.

The new NN potentials $[25,26]$ were employed by LENPIC members in few-nucleon and many nucleon systems [27-29]. Results for several few-nucleon observables obtained with the NN potentials alone show clear discrepancies between theoretical predictions and experimental data. These discrepancies are much larger than the estimated truncation uncertainty and agree with the expected magnitude of 3NF contributions. Unfortunately, the implementation of a consistent regulator in coordinate space proved to be very difficult for the $3 \mathrm{~N}$ potentials beyond N2LO, so in Ref. [31] the consistent investigation of nucleon-deuteron scattering as well as ground and low-lying excited states of nuclei with $A \leq 16$ was performed only up to N2LO. One of the important questions posed in that study was the determination of the lowenergy constants $c_{D}$ and $c_{E}$, specifying the 3NF at N2LO. On top of the experimental triton binding energy, which provides a connection between these two parameters, an observable from nucleon-deuteron scattering is necessary to fix the $c_{D}$ and $c_{E}$ values. The very precise experimental data for the proton-deuteron differential cross section at the proton laboratory energy of $70 \mathrm{MeV}$ from Ref. [32] was chosen, taking into account the theoretical uncertainty from the truncation of the chiral expansion.

Calculations described in Ref. [33] showed additionally that combining N4LO NN potentials with the N2LO 3NF regularized in the same way leads to similar 3NF effects in nucleondeuteron scattering as found with semi-phenomenological nuclear forces. In particular, the 
application of these forces did not explain the low energy $A_{y}$ puzzle [33]. For a detailed comparison of these chiral potential based predictions to data we refer the reader to Ref. [33].

The latest generation of the chiral $2 \mathrm{~N}$ potentials, introduced in Ref. [30], employs a momentum-space version of the local regulator. These potentials developed up to the so-called "N4LO+" version are currently the most precise chiral interactions on the market. They reach at least the same accuracy in reproducing NN scattering data below the pion production threshold as the phenomenological high-precision potentials but with a significantly smaller number of adjustable parameters.

In a very recent paper [34] the study of Ref. [31] was updated and nucleon-deuteron scattering observables were analyzed using the newest chiral NN potentials of Ref. [30] in combination with consistently regularized N2LO 3N forces. The former way of estimation of truncation uncertainties was replaced by a Bayesian approach. The results for elastic nucleon-deuteron scattering observables confirmed predictions based on the coordinate-space version of the local regulator and agree within errors with experimental data. Additionally they indicated that a precise description of neutron-deuteron scattering below pion production threshold would require $3 \mathrm{~N}$ forces (at least) at the N4LO level, see [35-37] for some work along this line.

In this contribution, in some measure by definition, we restrict ourselves to the nuclear forces developed within the LENPIC project. But we are of course aware of the important contributions made by other groups. Here we only mention the Moscow (Idaho)-Salamanca group [38] results, the nuclear forces constructed by Piarulli et al. [39, 40], by Ekström et al. $[41,42]$ and a very recent family of potentials intended for nuclear structure calculations [43]. For a more general discussion of the recent chiral models of the nuclear interactions we refer the reader to Ref. [34].

\section{Electroweak processes}

Electroweak processes are also of interest to the LENPIC project. They require single-nucleon and many-nucleon electromagnetic and axial current operators, which are derived consistently with the nuclear forces. Although electromagnetic and axial currents have been worked out in chiral effective field theory completely up through N3LO [44-47] using the method of unitary transformation and employing dimensional regularization for loop integrals, they are not yet regularized and thus cannot be used in practical calculations together with the nuclear forces employing the coordinate- or momentum-space regularization.

That is why we studied various electroweak processes: muon capture on ${ }^{3} \mathrm{He}$ and ${ }^{3} \mathrm{H}[48$, 49], (anti)neutrino scattering off ${ }^{3} \mathrm{He}$ and ${ }^{3} \mathrm{H}[50,51]$ and pion radiative capture in ${ }^{3} \mathrm{He}$ and ${ }^{3} \mathrm{H}[52]$ exactly in the same framework as electron scattering on ${ }^{3} \mathrm{He}$ and ${ }^{3} \mathrm{H}$ and photodisintegration of ${ }^{3} \mathrm{He}$ and ${ }^{3} \mathrm{H}$ [53], using the semi-phenomenological AV18 [2] potential and the single nucleon current, including additionally in some cases $3 \mathrm{NF}$ and $2 \mathrm{~N}$ current effects. We not only provided realistic predictions for all the above mentioned reactions but argued that all these processes should be studied within LENPIC using the consistent nuclear forces and current operators. Some of the calculated observables will be used to fix the low-energy constants in the chiral electromagnetic and axial currents.

The chiral electroweak current operators are generally not expected to be used to analyze results of experiments, where electromagnetic and weak probes transfer a lot of energy and large momenta to a nuclear system. (See, however, recent predictions for elastic electrondeuteron scattering in Ref. [54].) That is why first of all we plan to apply chiral currents to low-energy photodisintegration processes and muon capture from the lowest atomic orbits, where the energy and momentum transfers are naturally limited. Calculations based on chiral forces and currents will be important for the results of electron scattering experiments planned 


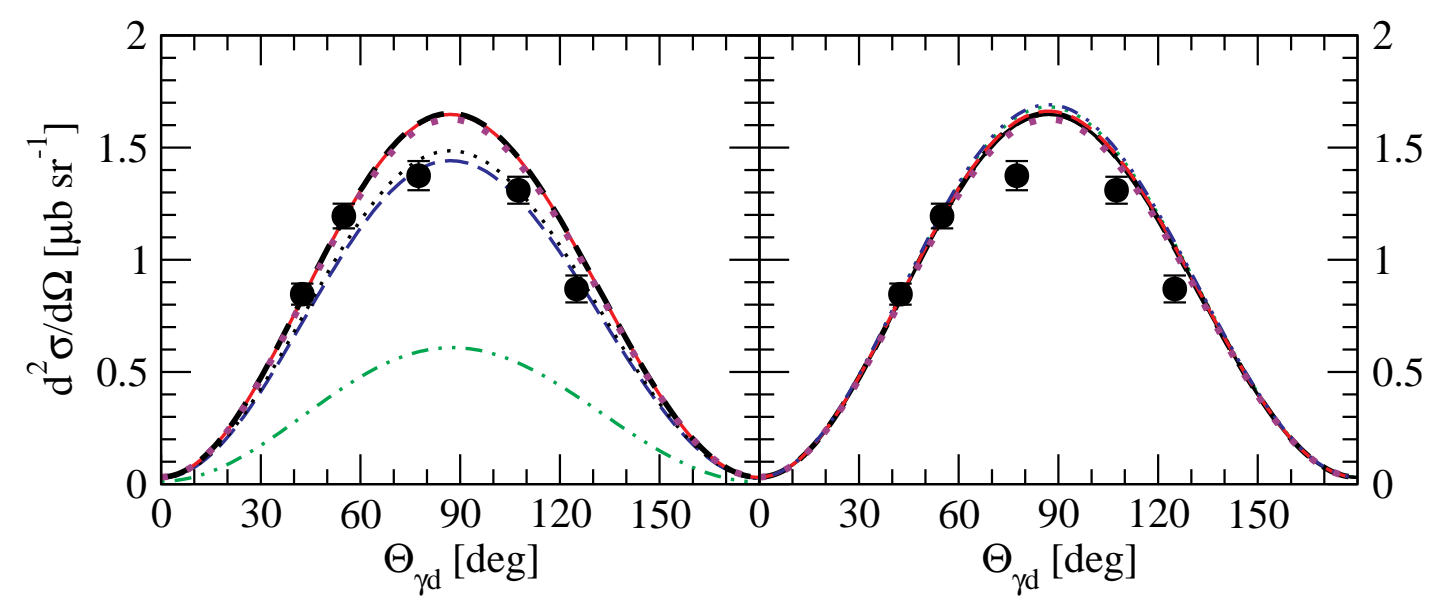

Figure 2: The differential cross section $d^{2} \sigma / d \Omega$ for the $n+d \rightarrow \gamma+{ }^{3} \mathrm{H}$ reaction at the neutron laboratory energy $E_{n}=9 \mathrm{MeV}$ as a function of the center-of-mass angle $\Theta_{\gamma d}$ between the initial deuteron and final photon momenta obtained with the chiral NN potential $[25,26]$ and the Siegert theorem [53]. In the left panel various lines show predictions from Ref. [57] with the fixed cut-off parameter $R=0.9 \mathrm{fm}$ at various orders of chiral expansions: LO (double-dotted-dashed green), NLO (dashed blue), N2LO (dotted black), N3LO (solid red) and N4LO (thick dashed black). In the right panel the lines show N4LO predictions for various cut-off parameter values $R$ : 0.8 $\mathrm{fm}$ (dotted green), $0.9 \mathrm{fm}$ (thick dashed black), $1.0 \mathrm{fm}$ (solid black), $1.1 \mathrm{fm}$ (dashed red) and $1.2 \mathrm{fm}$ (double-dotted-dashed blue). In both cases we added our predictions based on the AV18 NN potential [2], represented in the two panels by the thick violet dotted curve. The data are from Ref. [58].

at the MESA accelerator in Mainz, Germany.

Despite the lack of the electroweak currents consistent with the newest chiral nucleonnucleon potentials, electromagnetic processes with real photons were studied in Ref. [55] using the Siegert theorem. In this way we could include implicitly certain types of manynucleon contributions in the nuclear current operator. The results obtained with the potentials employing the local coordinate-space regularization turned out to be very promising, revealing a very weak dependence of predictions on the cutoff parameter and providing a good data description.

As an example, we show in Fig. 2 our predictions from Ref. [57] for the differential cross section $d^{2} \sigma / d \Omega$ for the $n+d \rightarrow \gamma+{ }^{3} \mathrm{H}$ reaction at the neutron laboratory energy $E_{n}=9$ $\mathrm{MeV}$. The left panel of Fig. 2 shows a fast convergence of the results with respect to the chiral order for the fixed regulator value of $R=0.9 \mathrm{fm}$ and the right panel demonstrates a weak dependence on the regulator value at N4LO. Additionally the N4LO results agree very well with the predictions based on the AV18 potential and, most importantly, with the data [58].

\section{Conclusion}

The reader should be aware that in this contribution we restricted ourselves to the the fewnucleon part of the LENPIC project. Equally important are the investigations of many-nucleon systems performed within LENPIC [28,31,60,61], which put the current models of the nuclear forces to stringent tests.

All the studies mentioned in this contribution yielded very promising results and demonstrate the high quality of the newest chiral NN potentials [30]. Also the studies including the 
consistently regularized N2LO 3N force are very encouraging [34]. It is, however, very clear that these efforts should be extended at least to N3LO, which requires inclusion of the consistently regularized $3 \mathrm{~N}$ force and many-nucleon current contributions [56,59]. Work along these lines is in progress by the LENPIC Collaboration.

\section{Acknowledgements}

This work is a part of the LENPIC project. The numerical calculations were partially performed on the supercomputer cluster of the JSC, Jülich, Germany.

Funding information This work was supported by the Polish National Science Centre under Grants No. 2016/22/M/ST2/00173 and 2016/21/D/ST2/01120 as well as by BMBF (contract No. 05P18PCFP1) and by DFG and NSFC (TRR 110).

\section{References}

[1] The LENPIC website, http://www.lenpic.org/.

[2] R. B. Wiringa, V. G. J. Stoks and R. Schiavilla, Accurate nucleon-nucleon potential with charge-independence breaking, Phys. Rev. C 51, 38 (1995), doi:10.1103/PhysRevC.51.38.

[3] R. Machleidt, High-precision, charge-dependent Bonn nucleon-nucleon potential, Phys. Rev. C 63, 024001 (2001), doi:10.1103/PhysRevC.63.024001.

[4] V. G. J. Stoks, R. A. M. Klomp, C. P. F. Terheggen and J. J. de Swart, Construction of high-quality NN potential models, Phys. Rev. C 49, 2950 (1994), doi:10.1103/PhysRevC.49.2950.

[5] W. Glöckle, H. Witała, D. Hüber, H. Kamada and J. Golak, The three-nucleon continuum: achievements, challenges and applications, Phys. Rep. 274, 107 (1996), doi:10.1016/0370-1573(95)00085-2.

[6] H. Witała, W. Glöckle, J. Golak, A. Nogga, H. Kamada, R. Skibiński and J. KurośŻołnierczuk, $N d$ elastic scattering as a tool to probe properties of $3 \mathrm{~N}$ forces, Phys. Rev. C 63, 024007 (2001), doi:10.1103/PhysRevC.63.024007.

[7] S. A. Coon, M. D. Scadron, P. C. McNamee, B. R. Barrett, D. W. E. Blatt and B. H. J. McKellar, The two-pion-exchange three-nucleon potential and nuclear matter, Nucl. Phys. A 317, 242 (1979), doi:10.1016/0375-9474(79)90462-7.

[8] B. S. Pudliner, V. R. Pandharipande, J. Carlson, S. C. Pieper and R. B. Wiringa, Quantum Monte Carlo calculations of nuclei with A < 7, Phys. Rev. C 56, 1720 (1997), doi:10.1103/PhysRevC.56.1720.

[9] R. V. Cadman et al., Evidence for a three-nucleon-force effect in proton-deuteron elastic scattering, Phys. Rev. Lett. 86, 967 (2001), doi:10.1103/PhysRevLett.86.967.

[10] B. v. Przewoski et al., Analyzing powers and spin correlation coefficients for $p+d$ elastic scattering at 135 and $200 \mathrm{MeV}$, Phys. Rev. C 74, 064003 (2006), doi:10.1103/PhysRevC.74.064003. 
[11] R. Bieber et al., Three-nucleon force and the $A_{y}$ puzzle in intermediate energy $\vec{p}+d$ and $\vec{d}+$ p elastic scattering, Phys. Rev. Lett. 84, 606 (2000), doi:10.1103/PhysRevLett.84.606.

[12] H. Witała, J. Golak, W. Glöckle and H. Kamada, Relativistic effects in neutron-deuteron elastic scattering, Phys. Rev. C 71, 054001 (2005), doi:10.1103/PhysRevC.71.054001.

[13] H. Witała, J. Golak, R. Skibiński, W. Glöckle, W. N. Polyzou and H. Kamada, Relativity and the low-energy nd $A_{y}$ puzzle, Phys. Rev. C 77, 034004 (2008), doi:10.1103/PhysRevC.77.034004.

[14] E. Epelbaum, A. Nogga, W. Glöckle, H. Kamada, U.-G. Meißner and H. Witała, Threenucleon forces from chiral effective field theory, Phys. Rev. C 66, 064001 (2002), doi:10.1103/PhysRevC.66.064001.

[15] D. R. Entem and R. Machleidt, Accurate charge-dependent nucleon-nucleon potential at fourth order of chiral perturbation theory, Phys. Rev. C 68, 041001 (2003), doi:10.1103/PhysRevC.68.041001.

[16] E. Epelbaum, W. Glöckle and U.-G. Meißner, The two-nucleon system at next-to-next-to-next-to-leading order, Nucl. Phys. A 747, 362 (2005), doi:10.1016/j.nuclphysa.2004.09.107.

[17] J. R. Bergervoet, P. C. van Campen, R. A. M. Klomp, J.-L. de Kok, T. A. Rijken, V. G. J. Stoks and J. J. de Swart, Phase shift analysis of all proton-proton scattering data below $T_{l a b}=350 \mathrm{MeV}$, Phys. Rev. C 41, 1435 (1990), doi:10.1103/PhysRevC.41.1435.

[18] V. G. J. Stoks, R. A. M. Klomp, M. C. M. Rentmeester and J. J. de Swart, Partial-wave analysis of all nucleon-nucleon scattering data below $350 \mathrm{MeV}$, Phys. Rev. C 48, 792 (1993), doi:10.1103/PhysRevC.48.792.

[19] V. Bernard, E. Epelbaum, H. Krebs and U.-G. Meißner, Subleading contributions to the chiral three-nucleon force: Long-range terms, Phys. Rev. C 77, 064004 (2008), doi:10.1103/PhysRevC.77.064004.

[20] V. Bernard, E. Epelbaum, H. Krebs and U.-G. Meißner, Subleading contributions to the chiral three-nucleon force. II. Short-range terms and relativistic corrections, Phys. Rev. C 84, 054001 (2011), doi:10.1103/PhysRevC.84.054001.

[21] J. Golak et al., A new way to perform partial-wave decompositions of few-nucleon forces, Eur. Phys. J. A 43, 241 (2010), doi:10.1140/epja/i2009-10903-6.

[22] R. Skibiński, J. Golak, K. Topolnicki, H. Witała, H. Kamada, W. Glöckle and A. Nogga, The Tucson-Melbourne three-nucleon force in the automatized partial-wave decomposition, Eur. Phys. J. A 47, 48 (2011), doi:10.1140/epja/i2011-11048-9.

[23] K. Hebeler, H. Krebs, E. Epelbaum, J. Golak and R. Skibiński, Efficient calculation of chiral three-nucleon forces up to $N^{3} L O$ for ab initio studies, Phys. Rev. C 91, 044001 (2015), doi:10.1103/PhysRevC.91.044001.

[24] H. Witała, J. Golak, R. Skibiński and K. Topolnicki, Calculations of three-nucleon reactions with $N^{3}$ LO chiral forces: achievements and challenges, J. Phys. G: Nucl. Part. Phys. 41, 094011 (2014), doi:10.1088/0954-3899/41/9/094011.

[25] E. Epelbaum, H. Krebs and U. -G. Meißner, Improved chiral nucleon-nucleon potential up to next-to-next-to-next-to-leading order, Eur. Phys. J. A 51, 53 (2015), doi:10.1140/epja/i2015-15053-8. 
[26] E. Epelbaum, H. Krebs and U.-G. Meißner, Precision nucleon-nucleon potential at fifth order in the chiral expansion, Phys. Rev. Lett. 115, 122301 (2015), doi:10.1103/PhysRevLett.115.122301.

[27] S. Binder et al., Few-nucleon systems with state-of-the-art chiral nucleon-nucleon forces, Phys. Rev. C 93, 044002 (2016), doi:10.1103/PhysRevC.93.044002.

[28] S. Binder et al., Few-nucleon and many-nucleon systems with semilocal coordinatespace regularized chiral nucleon-nucleon forces, Phys. Rev. C 98, 014002 (2018), doi:10.1103/PhysRevC.98.014002.

[29] H. Witała et al., Role of the total isospin 3/2 component in three-nucleon reactions, FewBody Syst. 57, 1213 (2016), doi:10.1007/s00601-016-1156-3.

[30] P. Reinert, H. Krebs and E. Epelbaum, Semilocal momentum-space regularized chiral two-nucleon potentials up to fifth order, Eur. Phys. J. A 54, 86 (2018), doi:10.1140/epja/i2018-12516-4.

[31] E. Epelbaum et al., Few- and many-nucleon systems with semilocal coordinate-space regularized chiral two- and three-body forces, Phys. Rev. C 99, 024313 (2019), doi:10.1103/PhysRevC.99.024313.

[32] K. Sekiguchi et al., Complete set of precise deuteron analyzing powers at intermediate energies: Comparison with modern nuclear force predictions, Phys. Rev. C 65, 034003 (2002), doi:10.1103/PhysRevC.65.034003.

[33] H. Witała et al., Application of semilocal coordinate-space regularized chiral forces to elastic Nd scattering and breakup, Few Body Syst. 60, 19 (2019), doi:10.1007/s00601-019$1485-0$.

[34] E. Epelbaum et al., Towards high-order calculations of three-nucleon scattering in chiral effective field theory (2019), arXiv:1907.03608.

[35] H. Krebs, A. Gasparyan and E. Epelbaum, Chiral three-nucleon force at $N^{4} L O$ I: Longestrange contributions, Phys. Rev. C 85, 054006 (2012), doi:10.1103/PhysRevC.85.054006.

[36] H. Krebs, A. Gasparyan and E. Epelbaum, Chiral three-nucleon force at $N^{4}$ LO II. Intermediate-range contributions, Phys. Rev. C 87, 054007 (2013), doi:10.1103/PhysRevC.87.054007.

[37] L. Girlanda, A. Kievsky and M. Viviani, Subleading contributions to the three-nucleon contact interaction, Phys. Rev. C 84, 014001 (2011), doi:10.1103/PhysRevC.84.014001.

[38] D. R. Entem, R. Machleidt and Y. Nosyk, High-quality two-nucleon potentials up to fifth order of the chiral expansion, Phys. Rev. C 96, 024004 (2017), doi:10.1103/PhysRevC.96.024004.

[39] M. Piarulli, L. Girlanda, R. Schiavilla, R. Navarro Pérez, J. E. Amaro and E. Ruiz Arriola, Minimally nonlocal nucleon-nucleon potentials with chiral two-pion exchange including $\Delta$ resonances, Phys. Rev. C 91, 024003 (2015), doi:10.1103/PhysRevC.91.024003.

[40] M. Piarulli, L. Girlanda, R. Schiavilla, A. Kievsky, A. Lovato, L. E. Marcucci, S. C. Pieper, M. Viviani and R. B. Wiringa, Local chiral potentials with $\Delta$-intermediate states and the structure of light nuclei, Phys. Rev. C 94, 054007 (2016), doi:10.1103/PhysRevC.94.054007. 
[41] A. Ekström et al., Accurate nuclear radii and binding energies from a chiral interaction, Phys. Rev. C 91, 051301 (2015), doi:10.1103/PhysRevC.91.051301.

[42] A. Ekström, G. Hagen, T. D. Morris, T. Papenbrock and P. D. Schwartz, $\Delta$ isobars and nuclear saturation, Phys. Rev. C 97, 024332 (2018), doi:10.1103/PhysRevC.97.024332.

[43] T. Hüther, K. Vobig, K. Hebeler, R. Machleidt, and R. Roth, Family of chiral two- plus threenucleon interactions for accurate nuclear structure studies (2019), arXiv:1911.04955.

[44] S. Kölling, E. Epelbaum, H. Krebs and U. -G. Meißner, Two-pion exchange electromagnetic current in chiral effective field theory using the method of unitary transformation, Phys. Rev. C 80, 045502 (2009), doi:10.1103/PhysRevC.80.045502.

[45] S. Kölling, E. Epelbaum, H. Krebs and U.-G. Meißner, Two-nucleon electromagnetic current in chiral effective field theory: One-pion exchange and short-range contributions, Phys. Rev. C 84, 054008 (2011), doi:10.1103/PhysRevC.84.054008.

[46] H. Krebs, E. Epelbaum and U.-G. Meißner, Nuclear electromagnetic currents to fourth order in chiral effective field theory, Few-Body Syst. 60, 31 (2019), doi:10.1007/s00601-0191500-5.

[47] H. Krebs, E. Epelbaum and U.-G. Meißner, Nuclear axial current operators to fourth order in chiral effective field theory, Ann. Phys. 378, 317 (2017), doi:10.1016/j.aop.2017.01.021.

[48] J. Golak, R. Skibiński, H. Witała, K. Topolnicki, A. E. Elmeshneb, H. Kamada, A. Nogga and L. E. Marcucci, Break-up channels in muon capture on ${ }^{3} \mathrm{He}$, Phys. Rev. C 90, 024001 (2014), doi:10.1103/PhysRevC.90.024001.

[49] J. Golak, R. Skibiński, H. Witała, K. Topolnicki, H. Kamada, A. Nogga and L. E. Marcucci, Muon capture on ${ }^{3}$ H, Phys. Rev. C 94, 034002 (2016), doi:10.1103/PhysRevC.94.034002.

[50] J. Golak, R. Skibiński, K. Topolnicki, H. Witała, A. Grassi, H. Kamada and L. E. Marcucci, Momentum space treatment of inclusive neutrino scattering off the deuteron and trinucleons, Phys. Rev. C 98, 015501 (2018), doi:10.1103/PhysRevC.98.015501.

[51] J. Golak, R. Skibinski, K. Topolnicki, H. Witala, A. Grassi, H. Kamada and L. E. Marcucci, From response functions to cross sections in neutrino scattering off the deuteron and trinucleons, Phys. Rev. C 100, 064003 (2019), doi:10.1103/PhysRevC.100.064003.

[52] J. Golak, R. Skibiński, K. Topolnicki, H. Witała, A. Grassi, H. Kamada, A. Nogga and L. E. Marcucci, Radiative pion capture in ${ }^{2} \mathrm{H},{ }^{3} \mathrm{He}$, and ${ }^{3} \mathrm{H}$, Phys. Rev. C 98, 054001 (2018), doi:10.1103/PhysRevC.98.054001.

[53] J. Golak, R. Skibiński, H. Witała, W. Glöckle, A. Nogga and H. Kamada, Electron and photon scattering on three-nucleon bound states, Phys. Rep. 415, 89 (2005), doi:10.1016/j.physrep.2005.04.005.

[54] A. A. Filin, V. Baru, E. Epelbaum, H. Krebs, D. Möller, and P. Reinert, Extraction of the neutron charge radius from a precision calculation of the deuteron structure radius, arXiv:1911.04877.

[55] R. Skibiński et al., Testing semilocal chiral two-nucleon interaction in selected electroweak processes, Phys. Rev. C 93, 064002 (2016), doi:10.1103/PhysRevC.93.064002. 
[56] E. Epelbaum, Towards high-precision nuclear forces from chiral effective field theory (2019), arXiv:1908.09349.

[57] R. Skibiński, J. Golak, K. Topolnicki, H. Witała, E. Epelbaum, H. Kamada, H. Krebs, U.G. Meißner and A. Nogga, Modern chiral forces applied to the nucleon-deuteron radiative capture, Few-Body Syst. 58, 28 (2017), doi:10.1007/s00601-016-1190-1.

[58] G. Mitev, P. Colby, N. R. Roberson, H. R. Weller and D. R. Tilley, Radiative neutron capture by deuterium, Phys. Rev. C 34, 389 (1986), doi:10.1103/PhysRevC.34.389.

[59] H. Krebs, Electroweak current operators in chiral effective field theory (2019), arXiv:1908.01538.

[60] P. Maris, Ab initio calculations of p-shell nuclei up to $N^{2} L O$ in chiral Effective Field Theory, J. Phys.: Conf. Ser. 1291, 012005 (2019), doi:10.1088/1742-6596/1291/1/012005.

[61] P. Maris et al., Properties of ${ }^{4} \mathrm{He}$ and ${ }^{6} \mathrm{Li}$ with improved chiral EFT interactions, EPJ Web Conf. 113, 04015 (2016), doi:10.1051/epjconf/201611304015. 